\title{
A CONSTRUCTION OF ERGODIC BMO FUNCTIONS
}

\author{
KARL PETERSEN ${ }^{1}$
}

\begin{abstract}
One can explicitly construct unbounded functions having bounded mean oscillation (and in fact vanishing mean oscillation) with respect to any given ergodic measure-preserving transformation of a Lebesgue space.
\end{abstract}

1. BMO and VMO. A function which on the average does not differ too much from its average is said to have bounded mean oscillation, abbreviated BMO. When $f$ is a real-valued function of a real variable, this condition is expressed by requiring that

$$
\sup _{I} \frac{1}{m(I)} \int_{I}\left|f-\frac{1}{m(I)} \int_{I} f d m\right| d m<\infty,
$$

where $m$ is Lebesgue measure and the supremum is taken over all finite intervals $I \subset \mathbf{R}$ [9], [7] (see also [13] and the references given there). For the case of an ergodic measure-preserving transformation $T$ of a probability space $(X, \mathscr{B}, \mu)$, an analogous definition has been given by Coifman and Weiss [3]: a measurable function $f$ on $X$ is in BMO if and only if

$$
\|f\|_{\mathrm{BMO}}=\sup _{m} \operatorname{ess} \sup _{x} \frac{1}{m} \sum_{k=0}^{m-1}\left|f\left(T^{k} x\right)-\frac{1}{m} \sum_{j=0}^{m-1} f\left(T^{j} x\right)\right|<\infty .
$$

As stated in [3], these ergodic BMO functions have at least some of the interesting properties of the classical BMO functions: for example, they satisfy a certain distribution-function inequality, they can be represented as sums of bounded functions and their conjugates, and they form the dual of the space $H^{1}$ of all functions whose maximal functions are integrable.

Sarason [15] has defined the subclass of functions of vanishing mean oscillation (VMO); a real-valued function is in VMO if its oscillation over short intervals is small:

$$
\lim _{\delta \rightarrow 0} \sup _{m(I)<\delta} \frac{1}{m(I)} \int_{I}\left|f-\frac{1}{m(I)} \int_{I} f d m\right| d m=0 .
$$

For ergodic flows the analogous definition is formulated easily, but there is a problem for the case of a single transformation $T: X \rightarrow X$. However, Sarason proved that VMO coincides with the closure in BMO of the bounded uniformly continuous functions. Now because of the theorem of Jewett [8] and Krieger [11],

Received by the editors October 31, 1977.

AMS (MOS) subject classifications (1970). Primary 28A65.

${ }^{1}$ Research supported in part by N.S.F. Grant MCS 76-05786.

(c) 1980 American Mathematical Society 0002-9939/80/0000-0359/\$02.75 
we may assume that $(X, T)$ is a strictly ergodic subshift. That is, there is a closed shift-invariant subset $Y$ of the compact metric space $A^{Z}$ consisting of all bilateral sequences on a finite alphabet $A=\left\{a_{1}, a_{2}, \ldots, a_{r}\right\}$ such that (1) every orbit in $Y$ is dense in $Y$, (2) there is a unique shift-invariant Borel probability measure $\nu$ on $Y$, and (3) $(X, \mathscr{B}, \mu, T)$ is measure-theoretically isomorphic to the system consisting of $Y$, the restriction to $Y$ of the shift homeomorphism $\sigma$, and the measure $\nu$. We assume then that $\left(X, \mathscr{B}_{,}, T\right)=\left(Y, \mathscr{B}_{Y}, \nu, \sigma\right)$, where $\mathscr{B}_{Y}$ is the Borel field of $Y$. Once a topology for $X$ is produced in this way, we may form the BMO closure of $\mathcal{C}(X)$, thus producing an ergodic version of VMO.

2. Characterization and construction of ergodic VMO functions. We can characterize the ergodic VMO space $\mathrm{cl}_{\mathrm{BMO}} \mathcal{C}(X)$ by means of the Cesàro averages

$$
M_{n} f(x)=\frac{1}{n} \sum_{k=0}^{n-1} f\left(T^{k} x\right)
$$

Since $(X, T)$ is strictly ergodic, $M_{n} f \rightarrow \int f d \mu$ uniformly on $X$ for each $f \in \mathcal{C}(X)$. Write $f \dot{\in} \mathcal{C}(X)$ in case $f$ coincides a.e. $d \mu$ with a continuous function on $X$, and define

$$
\Re_{0}=\left\{f \in L^{1}(X, \mu): f-f T \dot{\in} \mathcal{C}(X) \text { and }\left\|M_{n} f-\int f d \mu\right\|_{\text {вMO }} \rightarrow 0\right\}
$$

and

$$
\Re=\left\{f \in L^{1}(X, \mu):\left\|M_{n} f-\int f d \mu\right\|_{\text {Вмо }} \rightarrow 0\right\} .
$$

Recall (see [5]) that the Hilbert transform $\tilde{f}(x)$ of a function $f \in L^{1}(X, \mu)$ is defined by

$$
\tilde{f}(x)=\lim _{n \rightarrow \infty} \sum_{\substack{k=-n \\ k \neq 0}}^{n} \frac{f\left(T^{k} x\right)}{k},
$$

the limit existing a.e. Let $\widetilde{C(X)}=\{\tilde{f}: f \in \mathcal{C}(X)\}$.

TheOrem 1. $e(X)+\widetilde{C(X)} \subset \mathfrak{M}_{0}=\operatorname{cl}_{\text {BMO }} e(X) \subset \mathfrak{N}$.

Proof. The first inclusion will follow if we can show that every function in $\mathcal{C}(X)$ is in $\Re_{0}$. Let $v \in \mathcal{C}(X)$ with $\int v d \mu=0$, so also $\int \tilde{v} d \mu=0$. Since $(v T)^{\sim}=\tilde{v} T$ and $\|\tilde{v}\|_{\mathrm{BMO}}<c\|v\|_{\infty}(\operatorname{see}[3]),\left\|M_{n} \tilde{v}\right\|_{\mathrm{BMO}}<c\left\|M_{n} v\right\|_{\infty} \rightarrow 0$. Also,

$$
\tilde{v}-\tilde{v} T=\sum_{k \neq 0,1} v T^{k}\left(\frac{1}{k}-\frac{1}{k-1}\right)+v T+v \dot{\epsilon} \mathcal{C}(X),
$$

since the series is absolutely and uniformly convergent.

To show that $\mathrm{cl}_{\text {BMO }} \mathcal{C}(X) \subset \mathfrak{N}_{0}$, note that if $f_{k} \in \mathcal{C}(X)$ and $f_{k} \rightarrow f$ in BMO, then already from the case $m=2$ in the definition of $\left\|f-f_{k}\right\|_{\text {BMO }}$ we must have

$$
0=\lim _{k} \frac{1}{2}\left\|(f-f T)-\left(f_{n}-f_{n} T\right)\right\|_{\infty} .
$$

Therefore $f-f T \dot{\in} \mathcal{C}(X)$. Since $\left\|M_{n} f-\int f d \mu\right\|_{\infty} \rightarrow 0$ for $f \in \mathcal{C}(X)$, it follows readily that $\left\|M_{n} f-\int f d \mu\right\|_{\text {BMO }} \rightarrow 0$ for $f \in \mathrm{cl}_{\text {BMO }} \mathcal{C}(X)$. 
Finally, if $f \in \Re_{0}$, then $f-f T, f T-f T^{2}, \ldots, f T^{n-2}-f T^{n-1} \dot{\in} \mathcal{C}(X)$, and summing and dividing by $n$ gives $f-M_{n} f \dot{\in} \mathcal{C}(X)$. Since $f-M_{n} f \rightarrow f-\int f d \mu$ in BMO, we must have $f \in \mathrm{cl}_{\text {BMO }} \mathcal{C}(X)$.

The space $T_{0}$ depends on the choice of the Jewett-Krieger representative of $T$, while $\mathfrak{N}$ does not. It would be interesting to determine the extent to which $\mathfrak{N}_{0}$ can vary with the choice of representative and to establish different characterizations of $\mathscr{R}$. Also, it is possible that $\mathcal{C}(X)+\widetilde{C(X)}=\Re_{0}$ in general.

The ergodic versions of BMO and VMO thus appear to be interesting spaces, but, unlike the classical case where examples are readily available, it is not immediately evident whether or not every system $(X, \mathscr{B}, \mu, T)$ has unbounded ergodic BMO and VMO functions. Indeed, only considering the case $m=2$ in (1.2) above shows that any such function $f$ must satisfy $f-f \circ T \in L^{\infty}$ (or $\mathcal{C}(X)$ ), $f \notin L^{\infty}$ (or $\left.\mathcal{C}(X)\right)$ and hence is likely to be rather complicated to describe. Because of the results of [2], it appears that if $f \in L^{1}$ and

$$
f^{*}(x)=\sup _{n>1}\left|(1 / n) \sum_{k=0}^{n-1} f\left(T^{k} x\right)\right|,
$$

then $\log f^{*} \in$ BMO. Here we show how to use Kakutani-Rokhlin towers to construct explictly unbounded ergodic BMO and VMO functions for each ergodic measure-preserving transformation $T$ of a Lebesgue space (separable nonatomic probability space) $(X, \mathscr{B}, \mu)$. We owe two of the key ideas behind the construction to earlier work of Anosov [1] and Conze [4], and we have also benefited from conversations with J. Brezin and B. Marcus.

THEOREM 2. Let $T: X \rightarrow X$ be an ergodic measure-preserving transformation of a Lebesgue space $(X, \mathscr{B}, \mu)$. Then $T$ admits unbounded ergodic VMO (and hence $B M O)$ functions.

3. A topological Kakutani-Rokhlin Lemma. The remainder of the paper consists of a proof of Theorem 2. We assume, as we may be taking a finite-entropy factor if necessary and using the Jewett-Krieger Theorem, that $(X, T)$ is a strictly ergodic subshift. A similar result to the following is found in [6].

LEMMA 1. If $(X, T)$ is a nonatomic strictly ergodic subsystem of the shift transformation on the space of sequences with entries from a finite alphabet, then for every integer $q>0$ it is possible to find an open-closed subset $E$ of $X$ such that $E$, $T E, \ldots, T^{q-1} E$ are pairwise disjoint and $E, T E, \ldots, T^{2 q-1} E$ cover $X$.

Proof. The proof of this statement is not much different from that of the well-known lemma of Rokhlin [14] and Kakutani [10]. First select a nonempty open-closed set $F$ such that $F, T^{-1} F, \ldots, T^{-q+1} F$ are pairwise disjoint. Such a set can be constructed by fixing $x \in X$, choosing disjoint open-closed neighborhoods $U_{i}$ of $T^{-i} x$ for $i=0, \ldots, q-1$, and letting $F=\cap_{i=0}^{q-1} T^{i} U_{i}$. Consider now the first-entry decomposition of $X$ with respect to $F$; that is, for some smallest integer $r>q, F \cup T^{-1} F \cup \cdots \cup T^{-r} F=X$, and if we let

$$
F_{0}=F, \quad F_{1}=T^{-1} F \backslash F_{0}, \ldots, F_{r}=T^{-r} F \backslash \bigcup_{i<r} F_{i}
$$


and $G_{k}=\left\{x \in F: T^{-k} x \in F\right.$ but $T^{-j} x \notin F$ for $\left.j<k\right\}$ for $k=1,2, \ldots, r+1$, then $X$ is decomposed into open-closed subsets as in the following diagram:

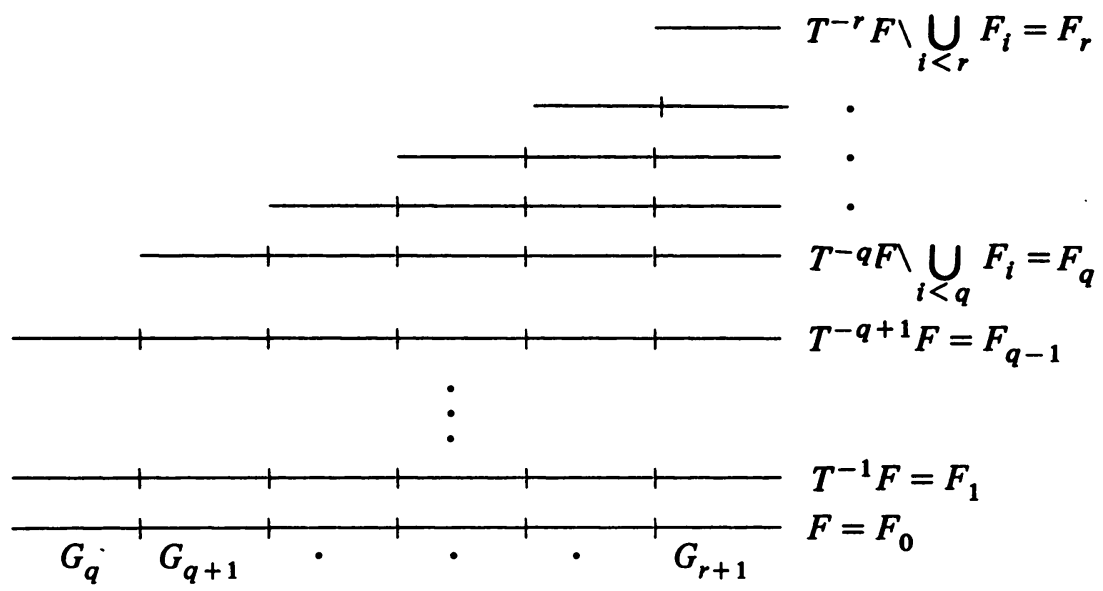

We now form the set $E$ by including from each column of the above diagram the top-most set and, working downwards, every $q$ th set, but not including any subsets of $F_{0}, F_{1}, \ldots$, or $F_{q-2}$. More precisely,

$$
E=\bigcup_{i=0}^{r-q+1} \bigcup_{j=0}^{[i / q]} T^{j q} T^{-q-i+1} G_{q+i}
$$

It is now clear that as we apply $T$, the various pieces of $E$ move downwards through the diagram in such a way that $E, T E, \ldots, T^{q-1} E$ are pairwise disjoint and $E \cup T E \cup \cdots \cup T^{2 q-1} E=X$,

4. Definition of $f$. The function $f \in$ BMO $\backslash L^{\infty}$ will be the sum of an infinite series, $f=\sum_{n=1}^{\infty} f_{n}$, where each $f_{n}$ is a continuous function on $X$ defined in terms of certain integers $q_{n}$ and $k_{n}$. The sequences will be generated inductively so as to satisfy

$$
\begin{gathered}
\sum_{n=1}^{\infty} 2^{n} \frac{k_{n+1}+1}{q_{n+1}}<\infty, \\
\sum_{n=1}^{\infty} \frac{q_{n}}{k_{n+1}}<\infty
\end{gathered}
$$

and

$$
\frac{1}{m} \sum_{j=0}^{m-1} f_{n}\left(T^{j} x\right)<2 \int_{X} f_{n} d \mu \text { for all } m>q_{n+1} \text {. }
$$

Condition (4.1) will guarantee that $\sum_{n=1}^{\infty} \int f_{n} d \mu<\infty$ and hence the series for $f$ converges a.e., and conditions (4.2) and (4.3) will be needed to verify (1.2).

To begin, using $\$ 3$ choose a nonempty open-closed subset $A_{1}$ of $X$ such that $A_{1} \cap T A_{1}=\varnothing$ and $A_{1} \cup T A_{1} \cup T^{2} A_{1} \cup T^{3} A_{1}=X$, let $q_{1}=k_{1}=2$, and define $f_{1} \equiv 1$ on $X$. Suppose that continuous nonnegative functions $f_{i}$, integers $q_{i}$ and $k_{i}<q_{i} / 2$, and open-closed sets $A_{i}$ have been defined for $i=1, \ldots, n$. For each 
$E \subset X$ with $\mu(E)>0$, denote by $T_{E}$ the first-return map to $E$; that is, if $n_{E}(x)=\inf \left\{n>0: T^{n} x \in E\right\}$, then

$$
T_{E} x=T^{n_{E}(x)} x \text { for } x \in E .
$$

Let us abbreviate $T_{A_{i}}$ by $T_{i}$. We now choose $k_{n+1}$ consistently with (4.2)-for example,

$$
k_{n+1}=2^{n+1} q_{n}
$$

will do-and then choose an even integer $q_{n+1}>q_{n}$ so as to satisfy both (4.1), say

$$
q_{n+1}>3^{n+1}\left(k_{n+1}+1\right) \text {, }
$$

and (4.3). Such a choice of $q_{n+1}$ is possible because $T$ is strictly ergodic and $f_{n}$ is continuous (see [12]). The integers $k_{n+1}$ and $q_{n+1}$ will then be used to define $f_{n+1}$ as follows. Select an open-closed subset $A_{n+1}$ of $A_{n}$ such that $A_{n+1}, T_{n} A_{n+1}, \ldots, T_{n+1}^{q_{n+1}-1} A_{n+1}$ are pairwise disjoint and $A_{n+1}, T_{n} A_{n+1}, \ldots, T_{n}^{2 q_{n+1}-1} A_{n+1}$ cover $A_{n}$, and define $f_{n+1}$ on $A_{n+1} \cup T_{n} A_{n+1}$ $\cup \cdots \cup T_{n}^{q_{n+1}-1} A_{n+1}$ to be the "spike function" which is 1 at the "center", $T_{n}^{q_{(n+1) / 2}-1} A_{n+1} \cup T_{n}^{q_{(n+1) / 2}} A_{n+1}$, and decreases in steps of $1 / k_{n+1}$ towards the two "ends" $A_{n+1}$ and $T_{n}^{q_{n+1}-1} A_{n+1}$. Since $k_{n+1}$ is small compared to $q_{n+1}, f_{n+1}$ takes the value 0 on most of the sets $T_{n}^{j} A_{n+1}, 0<j<q_{n+1}-1$. In analytic terms, $f_{n+1}$ is defined by

$$
f_{n+1}\left|T_{n}^{q_{n+1} / 2+j} A_{n+1}=f_{n+1}\right| T_{n}^{q_{n+1} / 2-j-1} A_{n+1} \equiv 1-\frac{j}{k_{n+1}} \quad \text { for } 0<j<k_{n+1},
$$

and

$$
f_{n+1} \mid T_{n+1}^{q_{n+1} / 2+j} A_{n+1} \equiv 0 \text { if }|j|>k_{n+1}+1 .
$$

The definition of $f_{n+1}$ is propagated forward to the rest of $X$ along the orbits: for each $x$ let $f_{n+1}(x)=f_{n+1}\left(T^{-k} x\right)$, where $k=\inf \left\{i>0: T^{-i} x \in \cup_{j=0}^{q_{n+1}-1} T_{n}^{j} A_{n+1}\right\}$. Then $f_{n+1}(x)=f_{n+1}(T x)$ unless $T x \in A_{n}$, in which case we still have

$$
\left|f_{n+1}(x)-f_{n+1}(T x)\right|<\frac{1}{k_{n+1}}
$$

In order to visualize this scheme of definition, note that $f_{n+1}$ has essentially the same graph on $A_{n}$ as on each of its copies $T_{n-1} A_{n}, T_{n-1}^{2} A_{n}, \ldots, T_{n-1}^{q_{n}-1} A_{n}$. This graph is repeated in each copy of $A_{n-1}$, and so forth up to $X$ itself. Also, $f_{n+1}$ is constant on each element of a certain partition of $X$ into finitely many open-closed subsets and therefore is continuous on $X$.

5. The integral of $f_{n+1}$. Note first that

$$
\int_{A_{n}} f_{n+1} d \mu=2 \sum_{j=1}^{k_{n+1}} \frac{j}{k_{n+1}} \mu\left(A_{n+1}\right)=\left(k_{n+1}+1\right) \mu\left(A_{n+1}\right) .
$$

Since $\int_{T_{n-1}^{j} A_{n}} f_{n+1} d \mu=\int_{A_{n}} f_{n+1} d \mu$ for $j=0, \ldots, q_{n}-1$, and

$$
\int_{T_{n=1}^{j}=1 A_{n} \backslash \cup_{i<j+1} T_{n-1}^{i} A_{n}} f_{n+1} d \mu<\int_{T_{n-1}^{j} A_{n} \backslash \cup_{\ll<} T_{n-1}^{i} A_{n}} f_{n+1} d \mu
$$


for $j=q_{n}-1, \ldots, 2 q_{n}-2$, it follows that

$$
\int_{A_{n}-1} f_{n+1} d \mu<2 q_{n} \int_{A_{n}} f_{n+1} d \mu \text {. }
$$

Similarly,

$$
\int_{A_{n-2}} f_{n+1} d \mu \leqslant 2 q_{n-1} \int_{A_{n-1}} f_{n+1} d \mu \leqslant 2^{2} q_{n-1} q_{n} \int_{A_{n}} f_{n+1} d \mu ;
$$

after a suitable number of repetitions of this type of estimate, we see that

$$
\begin{aligned}
\int_{X} f_{n+1} d \mu & \leqslant 2^{n} q_{1} q_{2} \cdots q_{n} \int_{A_{n}} f_{n+1} d \mu<2^{n} q_{1} q_{2} \cdots q_{n}\left(k_{n+1}+1\right) \mu\left(A_{n+1}\right) \\
& \leqslant 2^{n} \frac{k_{n+1}+1}{q_{n+1}} .
\end{aligned}
$$

By (4.1), $\sum_{n=1}^{\infty} \int_{X} f_{n} d \mu<\infty$, and this implies that $\sum_{n=1}^{\infty} f_{n}(x)$ converges a.e. Therefore $f=\sum_{n=1}^{\infty} f_{n}$ is well defined.

6. Proof that $f \in \mathrm{BMO} \backslash L^{\infty}$. From the construction of $f$ it is clear that for each $n=1,2, \ldots$ there is a set of positive measure on which $f_{1} \equiv f_{2} \equiv \cdots \equiv f_{n} \equiv 1$. Then clearly $f=\sum_{n=1}^{\infty} f_{n} \notin L^{\infty}$.

In order to verify (1.2), fix $m=1,2, \ldots$ and let

$$
\theta_{n}(x)=\frac{1}{m} \sum_{k=0}^{m-1}\left|f_{n}\left(T^{k} x\right)-\frac{1}{m} \sum_{j=0}^{m-1} f_{n}\left(T^{j} x\right)\right| \text { for } n=1,2, \ldots
$$

Then

$$
\frac{1}{m} \sum_{k=0}^{m-1}\left|f\left(T^{k} x\right)-\frac{1}{m} \sum_{j=0}^{m-1} f\left(T^{j} x\right)\right| \leqslant \sum_{n=1}^{\infty} \theta_{n}(x) .
$$

We split up the latter sum into three parts, consisting of the $n$ 's for which $m$ looks large, small, and moderate, respectively:

$$
\sum_{n=1}^{\infty} \theta_{n}(x)=\sum_{m>q_{n+1}} \theta_{n}(x)+\sum_{m<q_{n-1}} \theta_{n}(x)+\sum_{q_{n-1}<m<q_{n+1}} \theta_{n}(x) .
$$

In order to estimate the first term, observe that for $m>q_{n+1}$ we have

$$
\theta_{n}(x)<2 \frac{1}{m} \sum_{k=0}^{m-1} f_{n}\left(T^{k} x\right)<4 \int_{X} f_{n} d \mu
$$

by (4.3); therefore

$$
\sum_{m>q_{n+1}} \boldsymbol{\theta}_{n}(x)<4 \sum_{n=1}^{\infty} \int_{X} f_{n} d \mu<\infty .
$$

For the second term, we use (4.6), which implies that $\left|f_{n}\left(T^{k} x\right)-f_{n}\left(T^{j} x\right)\right|<$ $q_{n-1} / k_{n}$ for $j, k=0,1, \ldots, m-1$ provided that $m<q_{n-1}$. This yields

$$
\sum_{m<q_{n-1}} \theta_{n}(x)<\sum_{m<q_{n-1}} \frac{1}{m} \sum_{k=0}^{m-1} \frac{1}{m} \sum_{j=0}^{m-1}\left|f_{n}\left(T^{k} x\right)-f_{n}\left(T^{j} x\right)\right|<\sum_{n=2}^{\infty} \frac{q_{n-1}}{k_{n}}<\infty
$$


by (4.2). Finally, the sum comprising the third term, $\Sigma_{q_{n-1}<m<q_{n+1}} \theta_{n}$, consists of exactly two terms, since $\left\{q_{n}\right\}$ is increasing. Each of these terms is at most $2\left\|f_{n}\right\|_{\infty}<2$, so the third term is bounded by 4 . Combining these results shows that

$$
\frac{1}{m} \sum_{k=0}^{m-1}\left|f\left(T^{k} x\right)-\frac{1}{m} \sum_{j=0}^{m-1} f\left(T^{j} x\right)\right|<4 \sum_{n=1}^{\infty} \int_{X} f_{n} d \mu+\sum_{n=1}^{\infty} \frac{q_{n-1}}{k_{n}}+4,
$$

a finite constant independent of $m$. This proves that $f \in$ BMO.

7. An unbounded ergodic VMO function. If $g=\sum_{n=1}^{\infty} f_{n} / n$, then as before $g \notin L^{\infty}$. However, since

$$
\lim _{n \rightarrow \infty}\left\|\sum_{k>n} \frac{f_{k}}{k}\right\|_{\mathrm{BMO}}=0
$$

$g$ is in the BMO closure of $\mathcal{C}(X)$.

\section{REFERENCES}

1. D. V. Anosov, On an additive functional homology equation connected with an ergodic rotation of the circle, Izv. Akad. Nauk SSSR 37 (1973) = Math USSR Izvestija 7 (1973), 1257-1271.

2. R. R. Coifman and R. Rochberg, Another characterization of BMO, preprint.

3. Ronald R. Coifman and Guido Weiss, Maximal functions and $\boldsymbol{H}^{\boldsymbol{P}}$ spaces defined by ergodic transformations, Proc. Nat. Acad. Sci. U.S.A. 70 (1973), 1761-1763.

4. Jean Pierre Conze, Equations fonctionelles et systèmes induits en theorie ergodique, Z. Wahrscheinlichkeitstheorie Verw. Gebiete 23 (1972), 75-82.

5. M. Cotlar, A unified theory of Hilbert transforms and ergodic theorems, Rev. Mat. Cuyana 1 (1955), 105-167.

6. Manfred Denker, Christian Grillenberger and Karl Sigmund, Ergodic theory on compact spaces, Springer-Verlag, New York, 1976.

7. C. Fefferman and E. M. Stein, $H^{p}$ spaces of several variables, Acta Math. 129 (1972), 137-193.

8. R. I. Jewett, The prevalence of uniquely ergodic systems, J. Math. and Mech. 19 (1970), 717-729.

9. F. John and L. Nirenberg, On functions of bounded mean ascillation, Comm. Pure Appl. Math. 14 (1961), 415-426.

10. S. Kakutani, Induced measure preserving transformations, Proc. Japan Acad. 19 (1943), 635-641.

11. W. Krieger, On unique ergodicity, Proc. Sixth Berkeley Sympos. on Math., Stat. and Prob., I, University of California Press, Berkeley, 1972, 327-346.

12. J. C. Oxtoby, Ergodic sets, Bull. Amer. Math. Soc. 58 (1952), 116-136.

13. K. E. Petersen, Brownian motion, Hardy spaces and bounded mean ascillation, Cambridge Univ. Press, Cambridge, 1977.

14. V. A. Rokhlin, A "general" measure-preserving transformation is not mixing, Dokl. Akad. Nauk SSSR 60 (1948), 349-351.

15. Donald Sarason, Functions of vanishing mean ascillation, Trans. Amer. Math. Soc. 207 (1975), 391-405.

Department of Mathematics, University of North Carolina, Chapel Hill, North Carolina 27514 